SECTION 4. Computer science, computer engineering and automation.

Anton Yurievich Potlov

Assistant of the department «Biomedical Engineering»

Tambov State Technical University, Russia

zerner@yandex.ru

\title{
DETECTION OF OPTICAL INHOMOGENEITIES OF BIOLOGICAL TISSUE USING TPSF CONFORMAL MAPPING
}

\begin{abstract}
A new method of direct (without inverse problem solution using methods of diffuse optical tomography) optical inhomogeneity, such as cysts, hematomas, tumors etc. detection in biological tissue. The basis of the method is preprocessing of the surface obtained from a collection of time-resolved data (TPSF) in the Cartesian frame with consecutive conformal mapping to two cylindrical surfaces.

Key words: diffuse optical tomography, late arriving photons, highly scattering media, conformal mapping.

Citation: Potlov AY (2014) DETECTION OF OPTICAL INHOMOGENEITIES OF BIOLOGICAL TISSUE USING TPSF CONFORMAL MAPPING. ISJ Theoretical \& Applied Science 9 (17): 31-33. doi: http://dx.doi.org/10.15863/TAS.2014.09.17.5
\end{abstract}

\section{УДК 535.361}

\section{ИСПОЛЬЗОВАНИЕ КОНФОРМНО-ОТОБРАЖЕННЫХ ВФРТ ДЛЯ ДЕТЕКТИРОВАНИЯ ОПТИЧЕСКИХ НЕОДНОРОДНОСТЕЙ БИОЛОГИЧЕСКИХ ТКАНЕЙ}

\begin{abstract}
Аннотация: Описан способ непосредственной (без решения обратной задачи методами диффузионной оптической томографии) регистрации оптических неоднородностей, таких как кисты, гематомы, опухоли и т.п. в биологической ткани. В основе предложенного способа лежит обработка трехмерной поверхности, полученной из совокупности разрешённых по времени данных (ВФРТ), в декартовой системе координат с последующим её конформным отображением в две поверхности в циилиндрической системе координат.
\end{abstract}

Ключевые слова: диффузионная оптическая томография, поздно пришедшие фотоны, сильно рассеивающие среды, конформное отображение.

Оптическое излучение, прошедшее через биологическую ткань содержит полезную информацию о форме, размерах, местоположении и оптических свойствах структурных компонентов ткани, т.е. о её оптическом строении [1-4]. Однако, для того чтобы получить томографические изображения (интерпретация этой полезной информации), необходимо решить обратную задачу распространения излучения применительно к конкретному исследуемому биологическому объекту [2-6]. Но из-за высокой концентрации рассеивающих частиц, отличающихся при этом формой, размерами, местоположением и т.п. процесс восстановления изображения в диффузионной оптической томографии (ДОТ) продолжителен по времени даже при использовании больших объемов оперативной памяти и скорости вычислений компьютерных систем, что является одной из ключевых проблем ДОТ [7]. В связи с этим активно разрабатываются [3, 6-10] упрощенные методы экспресс регистрации неоднородностей в биологических тканях. Эти методы, как правило, основаны либо на замене решения обратной задачи анализом формы ВФРТ, либо на серьёзных допущениях при решении обратной задачи. 
Целью данной работы является повышение точности метода [7] регистрации неоднородностей в сильно рассеивающих средах без решения обратной задачи.

Непосредственное детектирование оптических неоднородностей, таких как кисты, гематомы, опухоли и т.п. можно осуществить с помощью визуализации конформноотображённых временных функций рассеяния точки (ВФРТ) [7], заключающейся в предварительной обработке $\mathrm{R}(\alpha, \mathrm{t})$ и последующем её представлении в виде трехмерных поверхностей в цилиндрической системе координат. Для этого у всех ВФРТ оставляются только части, соответствующие поздно пришедшим фотонам (ППФ) [7]:

$$
\mathrm{R}_{\mathrm{p}}\left(\alpha, \mathrm{t}_{3}\right)=\mathrm{R}(\alpha, \mathrm{t}) \backslash \mathrm{R}\left(\alpha, \mathrm{t}_{2}\right),
$$

где $\mathrm{t}_{2}=n, 2 n, \ldots, \mathrm{T}_{\text {isot }}$ и $\mathrm{t}_{3}=\mathrm{T}_{\text {isot }}, \mathrm{T}_{\text {isot }}+n, \ldots, T . \mathrm{T}_{\text {isot }}-$ минимальное время, прошедшее с момента падения импульса на объект до момента достижения центра диффузии. Затем производится нормировка $\mathrm{R}_{\mathrm{p}}\left(\alpha, \mathrm{t}_{3}\right)$ относительно $\mathrm{R}_{\mathrm{p}}\left(\frac{360^{\circ}}{N}, \mathrm{t}_{3}\right)$ :

$$
\mathrm{R}_{\mathrm{n}}\left(\alpha, \mathrm{t}_{3}\right)=\frac{\mathrm{R}_{\mathrm{p}}\left(\alpha, \mathrm{t}_{3}\right)}{\mathrm{R}_{\mathrm{p}}\left(\frac{360^{\circ}}{N}, \mathrm{t}_{3}\right)},
$$

при этом для снижения негативного воздействия фоновых шумов, и как следствия из этого повышения точности регистрации неоднородностей, каждая ВРФТ аппроксимируется прямой линией. Поскольку фоновые шумы, возникающие при получении сигнала в ДОТ, не подчиняются закону нормального распределения для аппроксимации целесообразно использовать биквадратный метод или метод наименьших модулей.

На основе $\mathrm{R}_{\mathrm{n}}\left(\alpha, \mathrm{t}_{3}\right)$ создаётся эталонная функция [7]:

$$
\mathrm{R}_{\mathrm{st}}\left(\alpha, \mathrm{t}_{3}\right)=\mathrm{R}_{\mathrm{n}}\left(\frac{360^{\circ}}{N}, \mathrm{t}_{3}\right) .
$$

Функция $\mathrm{R}_{\mathrm{n}}\left(\alpha, \mathrm{t}_{3}\right)$ видоизменяется $\mathrm{c}$ учетом дополнительного коэффициента отображения, K, следующим образом:

$$
\mathrm{R}_{\mathrm{k}}\left(\alpha, \mathrm{t}_{3}\right)=\left\{\begin{array}{l}
1, \mathrm{R}_{\mathrm{n}}\left(\alpha, \mathrm{t}_{3}\right)=1 \\
1+\left(\mathrm{R}_{\mathrm{n}}\left(\alpha, \mathrm{t}_{3}\right)-1\right) \cdot \mathrm{K}, \mathrm{R}_{\mathrm{n}}\left(\alpha, \mathrm{t}_{3}\right) \neq 1
\end{array} .\right.
$$

При этом $\mathrm{K}$ задаётся пользователем и может быть любым действительным числом, кроме нуля [7].

Функция $\mathrm{R}_{\mathrm{st}}\left(\alpha, \mathrm{t}_{3}\right)$, а также в однородном случае функция $\mathrm{R}_{\mathrm{k}}\left(\alpha, \mathrm{t}_{3}\right)$ равны единице при любых $\alpha, \mathrm{t}_{3}$ и $\mathrm{K}$ из области допустимых значений [7]. Для их визуализации в виде трехмерных поверхностей в цилиндрической системе координат производится преобразование координат:

$$
\left\{\begin{array}{l}
\mathrm{q}=\sqrt{\alpha^{2}+\mathrm{t}_{3}^{2}} \\
\psi=\operatorname{arctg}\left(\frac{\alpha}{\mathrm{t}_{3}}\right)
\end{array}\right.
$$

В результате отображения получаются функции $\mathrm{R}_{s t}^{*}(q, \psi)$ и $\mathrm{R}_{\mathrm{k}}^{*}(q, \psi)$ :

$$
\begin{aligned}
& \mathrm{R}_{\mathrm{st}}\left(\alpha, \mathrm{t}_{3}\right) \rightarrow \mathrm{R}_{\mathrm{st}}^{*}(q, \psi), \\
& \mathrm{R}_{\mathrm{k}}\left(\alpha, \mathrm{t}_{3}\right) \rightarrow \mathrm{R}_{\mathrm{k}}^{*}(q, \psi),
\end{aligned}
$$

представляющие собой две совпадающие цилиндрические поверхности (рис. 1a).

В неоднородном случае $\mathrm{R}_{\mathrm{k}}\left(\alpha, \mathrm{t}_{3}\right)$ будет равна единице только при $\alpha=\frac{360^{\circ}}{N}$ и любом допустимом $\mathrm{t}_{3}$. Для других значений $\alpha$, т.е. других ВФРТ, значение функции $\mathrm{R}_{\mathrm{k}}\left(\alpha, \mathrm{t}_{3}\right)$ будет отличаться от единицы в большую или меньшую сторону в зависимости от типа неоднородности (поглощающая, рассеивающая), её местоположения, размера и значения 
коэффициента $\mathrm{K}$. При преобразовании $\mathrm{R}_{\mathrm{k}}\left(\alpha, \mathrm{t}_{3}\right)$ к функции (1) получится неровная цилиндрическая поверхность (рис. 1б), причем по характеру её искривления которой, можно судить о размере и местоположении неоднородности [7].

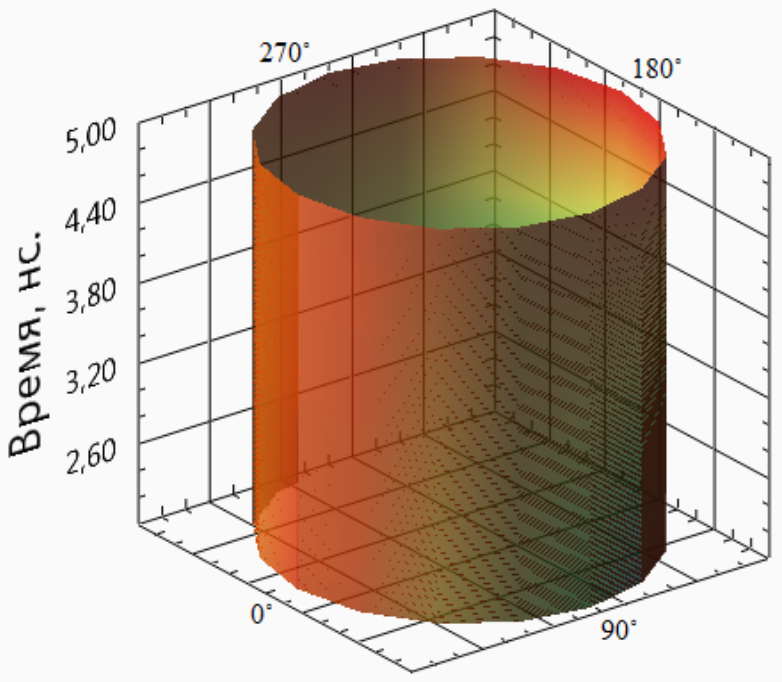

(a)

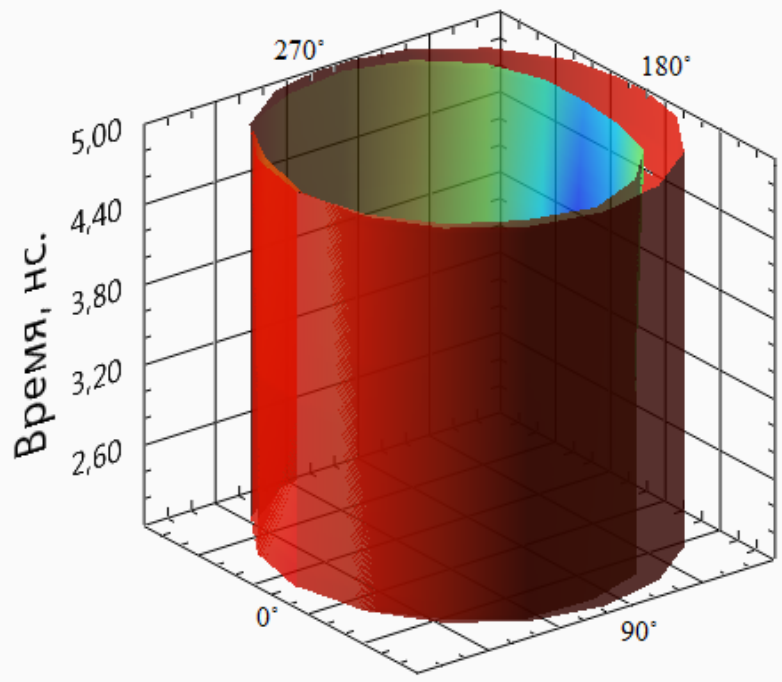

(б)

Рисунок 1. Трехмерное представление ППФ для однородного (а) и неоднородного (б) случаев, коэффициент отображения $K=-4$.

\section{References:}

1. Dehghani H, Srinivasan S, Pogue B, Gibson A (2009) Numerical modelling and image reconstruction in diffuse optical tomography. Phil. Trans. R. Soc. A., 367. doi: http://dx.doi.org/10.1098/rsta.2009.0090

2. Patterson M, Chance B, Wilson B (1989) Time resolved reflectance and transmittance for the noninvasive measurement of tissue optical properties. Appl. Opt. Vol. 28, pp. 23312336.

3. Zimnyakov DA, Tuchin VV (2002) Optical tomography of tissues. Quantum Electronics. Vol. 32. No.10, pp. 849-867.

4. Schweiger M, Arridge SR (1999) Application of Temporal filters to time-Resolved Data in Optical Tomography. Phys. Med. Biol. 1999. Vol. 44, pp.1699-1717.

5. Arridge SR, Cope M, Delpy DT (1992) Theoretical basis for the determination of optical pathlengths in tissue: temporal and frequency analysis. Physics in Medicine and Biology. 1992. Vol. 37. No.7, pp.1532-1560.

6. Proskurin SG (2011) Using late arriving photons for diffuse optical tomography of biological objects. Quantum Electron. Vol. 41 (5), pp. 402-406.

7. Potlov AYu, Proskurin SG, Frolov SV (2014) Three-dimensional representation of late arriving photons for the detection of inhomogeneous in diffuse optical tomography. Quantum Electronics. 2014. Vol. 44. No.2, pp. 174-181.

8. Proskurin SG, Potlov AYu, Frolov SV (2013) Detection of an absorbing heterogeneity in a biological object during recording of scattered photons. Biomedical Engineering. 2013. Vol. 46. No.6, pp.219-223.

9. Proskurin SG, Potlov AYu (2013) Early- and late-arriving photons in diffuse optical tomography. Photonics \& Lasers in Medicine. Vol.2. Iss.2, pp. 139-146. doi: http://dx.doi.org/10.1515/plm-2013-0003

10. Riley J, Amyot F, Pohida T, et al. (2012) A hematoma detector-a practical application of instrumental motion as signal in near infra-red imaging. Biomedical Optics Express. Vol. 3. No. 1, pp.192-205. 